\title{
A combined high-sugar and high-saturated-fat dietary pattern is associated with more depressive symptoms in a multi-ethnic population: the HELIUS (Healthy Life in an Urban Setting) study
}

\author{
Esther Vermeulen ${ }^{1, *}$, Karien Stronks ${ }^{1}$, Marieke B Snijder ${ }^{1,2}$, Aart H Schene ${ }^{3,4,5}$, Anja Lok ${ }^{3}$, \\ Jeanne $\mathrm{H}$ de Vries ${ }^{6}$, Marjolein Visser 7,8 , Ingeborg A Brouwer ${ }^{7}$ and Mary Nicolaou ${ }^{1}$ \\ 'Department of Public Health, Academic Medical Centre, University of Amsterdam, Amsterdam Public Health \\ Research Institute, Meibergdreef 9, 1105 AZ Amsterdam, The Netherlands: ${ }^{2}$ Department of Clinical Epidemiology, \\ Biostatistics and Bioinformatics, Academic Medical Centre, University of Amsterdam, Amsterdam, The Netherlands: \\ ${ }^{3}$ Program for Mood Disorders, Department of Psychiatry, Academic Medical Centre, University of Amsterdam, \\ Amsterdam, The Netherlands: ${ }^{4}$ Department of Psychiatry, Radboud University Medical Centre, Niimegen, The \\ Netherlands: ${ }^{5}$ Donders Institute for Brain, Cognition and Behaviour, Radboud University Nijmegen, Nijmegen, The \\ Netherlands: ${ }^{6}$ Division of Human Nutrition, Wageningen University, Wageningen, The Netherlands: ${ }^{7}$ Department of \\ Health Sciences, Faculty of Earth \& Life Sciences, Vrije Universiteit Amsterdam, Amsterdam Public Health Research \\ Institute, The Netherlands: ${ }^{8}$ Department of Nutrition and Dietetics, Internal Medicine, VU University Medical Centre, \\ Amsterdam Public Health Research Institute, Amsterdam, The Netherlands
}

Submitted 10 November 2016: Final revision received 30 May 2017: Accepted 1 June 2017: First published online 20 July 2017

\begin{abstract}
Objective: To identify a high-sugar (HS) dietary pattern, a high-saturated-fat (HF) dietary pattern and a combined high-sugar and high-saturated-fat (HSHF) dietary pattern and to explore if these dietary patterns are associated with depressive symptoms.

Design: We used data from the HELIUS (Healthy Life in an Urban Setting) study and included 4969 individuals aged 18-70 years. Diet was assessed using four ethnic-specific FFQ. Dietary patterns were derived using reduced rank regression with mono- and disaccharides, saturated fat and total fat as response variables. The nine-item Patient Health Questionnaire (PHQ-9) was used to assess depressive symptoms by using continuous scores and depressed mood (identified using the cut-off point: PHQ-9 sum score $\geq 10$ ).

Setting: The Netherlands.

Results: Three dietary patterns were identified; an HSHF dietary pattern (including chocolates, red meat, added sugars, high-fat dairy products, fried foods, creamy sauces), an HS dietary pattern (including sugar-sweetened beverages, added sugars, fruit (juices)) and an HF dietary pattern (including high-fat dairy products, butter). When comparing extreme quartiles, consumption of an HSHF dietary pattern was associated with more depressive symptoms (Q1 $v$. Q4: $\beta=0 \cdot 18,95 \%$ CI $0.07,0.30, P=0.001)$ and with higher odds of depressed mood (Q1 $v$. Q4: $\mathrm{OR}=2 \cdot 36,95 \%$ CI 1.19, 4.66, $P=0.014)$. No associations were found between consumption of the remaining dietary patterns and depressive symptoms.

Conclusions: Higher consumption of an HSHF dietary pattern is associated with more depressive symptoms and with depressed mood. Our findings reinforce the idea that the focus should be on dietary patterns that are high in both sugar and saturated fat.
\end{abstract}

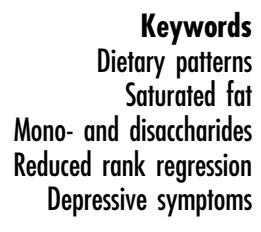

Keywords

Dietary patterns

Saturated fat

Mono- and disaccharides

Depressive symptoms
Depression is a mental disorder that is pervasive in the world and a major contributor to the global burden of disease $^{(1)}$. In the Netherlands, ethnic minority groups have a higher depression prevalence compared with the Dutch host population, with the highest prevalence among Turkish and Moroccan ethnic groups ${ }^{(2,3)}$. Because depression has a negative impact on people's functioning and is often recurrent or chronic, prevention of depression is a high priority in public health globally.

There is a growing body of evidence that diet may contribute to preventing depressive symptoms ${ }^{(4,5)}$. Healthy dietary patterns, including foods that are high in fibre (such 
as vegetables, fruits and whole grains), may be associated with lower depressive symptoms ${ }^{(6-8)}$. In contrast, unhealthy Western dietary patterns are implicated to be associated with depressive symptoms, although the evidence is equivocal $^{(9-14)}$. This could be due to differences in the use of dietary measurements or food group classification. Furthermore, there are differences in the measurement of depressive symptoms as some studies used self-reported scales (e.g. CES-D (Center for Epidemiologic Studies Depression Scale), PHQ-9 (nine-item Patient Health Questionnaire), GHQ (General Health Questionnaire)) whereas other studies used a clinical diagnosis for depression. Therefore, more studies are needed to allow a robust interpretation of the results ${ }^{(7)}$. Foods that are characteristic of a Western dietary pattern have high levels of refined carbohydrates and saturated fat. In a study by SanchezVillegas et al. a positive association was found between the intake of saturated fat and depressive symptoms ${ }^{(15)}$. Four other studies investigating the intake of sugar in relation to depressive symptoms, by studying sugar-sweetened beverages $^{(16)}$, high-energy sweet foods ${ }^{(17)}$ and the glycaemic index ${ }^{(18,19)}$ and depressive symptoms, found a significant positive association ${ }^{(16-19)}$. However, translating these findings to dietary advice is not straightforward. Restriction of one macronutrient implies an increased intake of another macronutrient to maintain energy balance, potentially leading to exacerbation of risk. For example, recent research revealed that substituting saturated fat by refined carbohydrates (as was done in recent decades in response to dietary guidelines limiting fat intake) showed no improvement or even worsening of the risk of $\mathrm{CVD}^{(20,21)}$. Because CVD and depression share common underlying mechanisms, such as increased production of proinflammatory cytokines and endothelial dysfunction ${ }^{(22,23)}$, it is of interest to further investigate the relative contribution of saturated fat and refined carbohydrates (specifically sugar) in overall dietary patterns to the risk of depressive symptoms.

One way to investigate this issue is by performing reduced rank regression (RRR) as this method derives overall dietary patterns and allows the investigation of the independent contributions of specific nutrients in these dietary patterns. The advantage of RRR is that it accounts for total dietary intake of the study participants and the derived dietary pattern is closely related to the outcome measure due to inclusion of intermediate response variables ${ }^{(24-26)}$.

The objective of our study was to identify a high-sugar (HS) dietary pattern, a high-saturated-fat (HF) dietary pattern and a combined high-sugar and high-saturated-fat (HSHF) dietary pattern and to explore if these dietary patterns are associated with depressive symptoms in a multi-ethnic Western population. In this population, heterogeneity is present due to differences in nutritional intake across ethnic groups, with ethnic minority populations consuming more high-sugar snacks but generally having a lower intake of saturated fat compared with the
Dutch ethnic group ${ }^{(27-29)}$. Consequently, we can capture the more extreme intakes of various dietary patterns, thereby being able to differentiate between our exposure: dietary patterns with diverse macronutrient compositions.

\section{Materials and methods}

\section{Participants and design}

The HELIUS (Healthy Life in an Urban Setting) study is a large, multi-ethnic cohort study which aims to unravel the causes of the unequal burden of disease and included Surinamese, Turkish, Moroccan and Dutch ethnic groups living in Amsterdam, the Netherlands ${ }^{(30)}$. Participants, aged 18-70 years, were randomly recruited, stratified by ethnicity, through the municipality registry of Amsterdam. Ethnicity was defined according to the country of birth of the participant as well as that of his/her parents ${ }^{(31)}$. We accounted for South-Asian Surinamese and African Surinamese separately due to differences in characteristics, which were classified according to self-reported ethnic origin. Data were collected by questionnaire (either selfadministered or by a trained ethnically matched interviewer) and a physical examination, which was conducted by trained research staff (research assistants and nurses) according to standardized protocols, at one of the local research sites of HELIUS ${ }^{(30)}$. Baseline data collection took place from 2011 until 2015 among nearly 25000 participants, and was carried out by the Academic Medical Centre and the Public Health Service of Amsterdam. The current study is a sub-sample of the HELIUS study and included 5188 participants who completed an additional ethnicspecific $\mathrm{FFQ}^{(32)}$. Participants were excluded when no data were available on depressive symptoms ( $n$ 26) or on demographics ( $n$ 193). All study protocols were approved by the Academic Medical Centre Ethical Review Board, and all participants provided written informed consent.

\section{Depressive symptoms assessment}

Depressive symptoms were assessed using the PHQ-9, a self-administered questionnaire that is used to estimate depressive symptoms over the previous two weeks and consists of nine items ${ }^{(33)}$. Response categories of the nine items vary from never (coded as ' 0 ') to nearly every day (coded as ' 3 ') with a total sum score ranging from 0 to 27. When only one of the nine items was missing, we imputed the mean of the other eight items. Within the HELIUS study population, the PHQ-9 was found to be measurement invariant regarding ethnicity and could therefore be validly used to compare depressive symptoms across ethnic groups (root-mean-squared error of approximation $=0.983$, comparative fit index =0.054; H Galenkamp, K Stronks, MB Snijder et al., unpublished results). We used the continuous PHQ-9 sum score as an outcome measure, hereafter referred to as 'depressive symptoms', as well as a dichotomous 
outcome variable (PHQ-9 sum score $\geq 10$ ), hereafter referred to as 'depressed mood'. This specific cut-off point has been previously validated ${ }^{(34,35)}$ and showed a sensitivity and specificity of 0.88 in primary-care patients ${ }^{(33)}$.

\section{Dietary intake assessment}

Four ethnic-specific FFQ, for Dutch, Surinamese, Turkish and Moroccan ethnic groups, were used to collect usual dietary intake information and were specially developed for the HELIUS study using an existing Dutch FFQ ${ }^{(36)}$. Each FFQ included questions on the portion size and frequency of approximately 200 food items eaten during the past month. For each FFQ, a nutrient database was constructed based on the Dutch Food Composition Table $2014^{(37)}$. Data on ethnic-specific foods were based on information from food packaging, international food composition data, data from recipe calculations and chemically analysed at Wageningen University by taking multiple samples of each food which were mixed for analysis; these samples were based on brand and different sale locations ${ }^{(36)}$. We used a single FFQ for the South-Asian Surinamese and African Surinamese group because of similarities in commonly eaten foods ${ }^{(38)}$.

\section{Dietary pattern analysis}

RRR is a statistical method that identifies patterns in a set of predictor variables (food groups) that explain the maximum variation in another set of variables known as response variables (nutrients or biomarkers), which are hypothesized to be intermediates between the food groups and a health outcome ${ }^{(25)}$. The mathematical foundations and technique of RRR are quite similar to principal component analysis. The major difference between RRR and principal component analysis is that the latter aims to explain the maximum variation in the food groups whereas RRR aims to explain the maximum variation in the intermediate response variables ${ }^{(26)}$. RRR starts from a linear function of the selected nutrients, called response scores, that will then be projected on to the space of the food groups to produce a factor score that is a linear function of the food groups. These factor scores (dietary pattern scores) were used for further analyses. As response variables, the nutrients mono- and disaccharides $(\mathrm{g} / \mathrm{d})$, saturated fatty acids $(\mathrm{g} / \mathrm{d})$ and total fatty acids $(\mathrm{g} / \mathrm{d})$ were used. All response variables were log-transformed because they were not normally distributed. As predictor variables, forty-six food groups (of which eight were ethnic-specific food groups) were composed based on nutrient profile, specific culinary use and, where relevant, ethnicity. For example, avocado was included as separate ethnic-specific group because it was assessed only in the Surinamese FFQ and the nutrient content of avocado (especially the fat content) is different compared with the foods that are included in the vegetables group. An overview of the composed food groups can be found in the online supplementary material, Supplemental Table 1. Dietary pattern scores were treated as continuous scores and as quartiles in all regression models. Higher scores and increasing quartiles reflect higher intakes of the food groups that are characteristic for the dietary patterns. Detailed information on the RRR procedure and the SAS code PLS is given elsewhere ${ }^{(25)}$.

\section{Measurement of covariates}

The following covariates were considered as potential confounders: age (years), sex, ethnicity (Dutch, SouthAsian Surinamese, African Surinamese, Turkish and Moroccan), marital status (married/registered partnership or living together, unmarried, divorced/separated or widowed), employment status (employed, not part of labour force (students, retirees, housewives/househusbands), unemployed (seeking work or incapacitated)), Dutch norm of physical activity (30 min of moderate exercise at least $5 \mathrm{~d}$ /week (yes or no)), smoking status (current smoker, never smoker, former smoker), energy intake (based on FFQ data in $\mathrm{kJ} / \mathrm{d}$ ), BMI based on measured height and weight $\left(\mathrm{kg} / \mathrm{m}^{2}\right)$, prevalent CVD according to the ROSE questionnaire (yes or no), selfreported hypertension (a reply of 'yes' to the question: 'Has a doctor or other health worker ever diagnosed you with hypertension?' Individuals were classified as having hypertension according to the WHO diagnostic criteria: systolic blood pressure $\geq 140 \mathrm{mmHg} /$ diastolic blood pressure $\geq 90 \mathrm{mmHg}$ or use of blood pressure-lowering medication (yes or no)) and self-reported type 2 diabetes mellitus (a reply of 'yes' to the question: 'Has a doctor or other health worker ever diagnosed you with diabetes?' Individuals were classified as having diabetes mellitus type 2 according to the WHO diagnostic criteria: fasting blood glucose $\geq 7 \mathrm{mmol} / \mathrm{l}$ and/or use of glucose-lowering medication (yes or no)).

\section{Statistical analysis}

Descriptive analyses were performed to get more insight into the baseline characteristics of the study population by using percentages for categorical variables and means and standard deviations for continuous variables. Multivariable linear regression models were applied to examine the cross-sectional association of continuous scores and quartiles of the HS dietary pattern, the HF dietary pattern and the HSHF dietary pattern with log-transformed depressive symptom sum scores as a continuous outcome measure. Additionally, logistic regression models were used to investigate the association between continuous scores and quartiles of all three dietary patterns and depressed mood as a dichotomous outcome (PHQ-9 sum score $\geq 10 ; P<0.05$ was considered as being statistically significant). Depressive symptom scores were log-transformed sum scores +1 , because the sum score was skewed to the right with a minimum of 0 . To check 
whether the diet-depression relationship was similar among ethnic groups and sex, the interaction between ethnicity and diet and between sex and diet was tested and was found to be significant for ethnicity and diet $(P<0.05)$. We chose to perform the regression analyses for the whole population including the interaction term of ethnicity $\times$ dietary pattern score, thereby maintaining the power of the sample size. Potential confounders (covariates that are listed previously) were included in the analyses when they changed the association between the dietary pattern score and depressive symptoms significantly $(P<0.05)$. Model 1 was adjusted for age, sex, ethnicity $\times$ dietary pattern score and ethnicity; model 2 was additionally adjusted for marital status, employment status, physical activity and energy intake; and in model 3 we additionally adjusted for BMI, CVD and diabetes. Smoking status and hypertension were not included in the final models because they did not change the dietdepression relationship significantly. Additionally, alcohol intake was not considered separately, as it was included as a predictor variable in the RRR analyses. To facilitate interpretations of the findings we back-transformed the $\beta$ coefficients using the following equation: $\left(e^{\beta}-1\right)$.

In sensitivity analyses, we excluded participants with extreme energy intakes ( $n$ 749) by using cut-off points according to Goldberg et ll $^{(39,40)}$. We applied this method because it takes account of the cut-off points on an individual level rather than on a population level. As the betweensubject variation in physical activity and energy intake is known to be high, applying the Goldberg cut-off may reduce bias ${ }^{(40)}$. Under-reporters were participants with an energy intake $<(B M R \times$ physical activity level $(\mathrm{PAL}))$ with PAL at a value of $\leq 0.87$ and over-reporters were participants with an energy intake $>\mathrm{BMR} \times \mathrm{PAL}$, with $\mathrm{PAL}>2 \cdot 75$. Furthermore, for the HS dietary pattern we further adjusted for dietary fibre intake and the healthier foods (fruit, low-fat dairy products) that were characteristic for the HS dietary pattern. Finally, we repeated RRR two times: first, we aimed to identify an HS dietary pattern by including mono- and disaccharides as single response variable; and second, we aimed to identify an HF dietary pattern by including saturated fatty acids as single response variable. By performing these additional dietary pattern analyses, we wanted to get more insight into whether it is possible to disentangle the effects of separate macronutrients into dietary patterns. The dietary pattern analyses were conducted in the statistical software package SAS version 9.4 and remaining statistical analyses were carried out using IBM SPSS Statistics version 21.

\section{Results}

\section{Baseline characteristics of the study population}

In total, we included 4969 participants with complete data for analyses. Baseline characteristics are presented in
Table 1 Baseline characteristics of the study population in the HELIUS (Healthy Life in an Urban Setting) study, Amsterdam, the Netherlands, 2011-2015

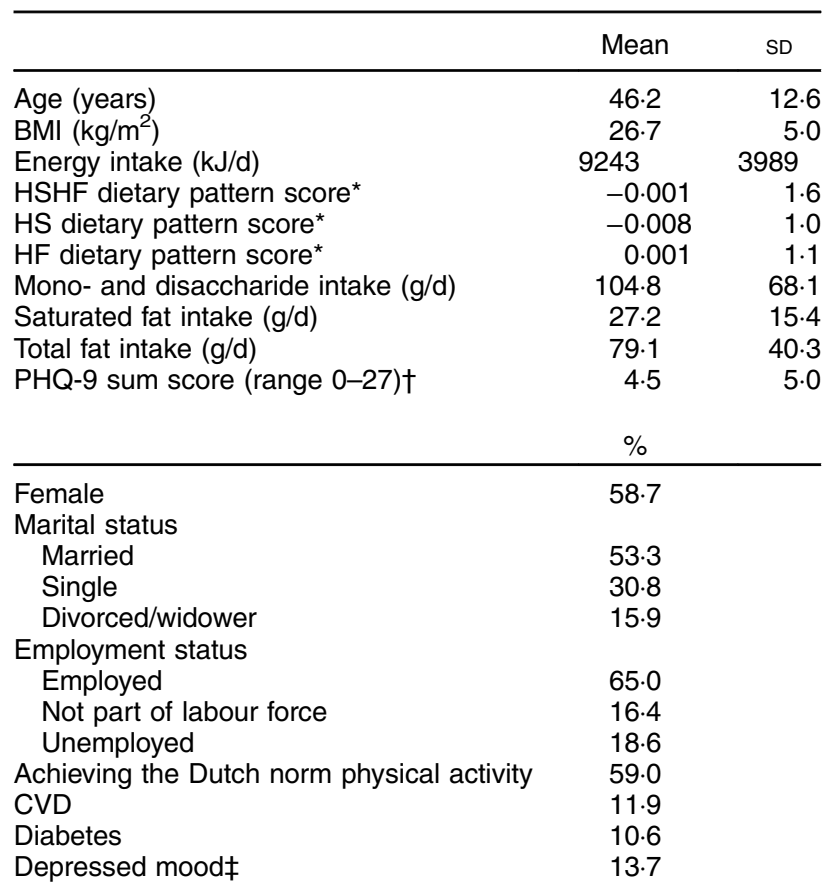

HSHF dietary pattern, combined high-sugar and high-saturated-fat; HS, high-sugar; HF, high-saturated-fat; PHQ-9, nine-item Patient Health Questionnaire.

*Higher dietary pattern scores indicate higher consumption of the food groups that are characteristic for the dietary patterns.

†A higher $\mathrm{PHQ}-9$ score means higher depressive symptoms.

†Depressed mood is defined as PHQ-9 sum score $\geq 10$.

Table 1. The mean BMI was $26.7 \mathrm{~kg} / \mathrm{m}^{2}$ and the mean energy intake was $9243 \mathrm{~kJ} / \mathrm{d}$ for the study population. On average, the study participants consumed $105 \mathrm{~g}$ monoand disaccharides/d and $27 \mathrm{~g}$ saturated fat/d. Finally, the mean depressive symptoms score was 5 .

\section{Dietary pattern analysis}

By using RRR, three dietary patterns were extracted. The first factor represented a dietary pattern that was positively associated with all three response variables and was labelled as 'HSHF dietary pattern' (high loadings of chocolates, sweets and pastries, red meat, added sugars, high-fat dairy products, fried foods, creamy sauces). The second factor represented a dietary pattern that was positively associated with mono- and disaccharides and negatively associated with saturated fatty acids and total fatty acids, and was labelled as 'HS dietary pattern' (high loadings of sugar-sweetened beverages, added sugars, fruit, fruit juices and very low loadings of red meat). The third dietary pattern was positively associated with saturated fatty acids and negatively associated with mono- and disaccharides and total fatty acids, being represented by high loadings of butter and high-fat dairy products and by very low loadings of vegetable oils, nuts and seeds, peanut butter and vegetables. This dietary pattern was labelled as 
Table 2 Food groups ${ }^{*}$ with their corresponding factor loadings derived by reduced rank regression with the percentage explained variation and correlation coefficients in the HELIUS (Healthy Life in an Urban Setting) study, Amsterdam, the Netherlands, 2011-2015

\begin{tabular}{|c|c|c|c|c|c|}
\hline Factor 1: HSHF dietary pattern & Load & Factor 2: HS dietary pattern & Load & Factor 3: HF dietary pattern & Load \\
\hline \multicolumn{6}{|l|}{ Positive loadings } \\
\hline Chocolates, sweets and pastries & 0.29 & Sugar sweetened beverages & 0.42 & Butter (spread and for cooking) & 0.32 \\
\hline Red meat & 0.26 & Natural fruit juices & 0.40 & High-fat dairy products & 0.27 \\
\hline Sugar, honey and jam & 0.23 & Fruit & 0.35 & & \\
\hline High-fat dairy products & 0.23 & Sugar, honey and jam & 0.32 & & \\
\hline Fried foods & 0.20 & Low-fat dairy products & 0.20 & & \\
\hline Creamy sauces & $0 \cdot 20$ & & & & \\
\hline Tivegauve ivaunins & & Red meat & -0.23 & Nuts and seeds & -0.33 \\
\hline & & & & Olive oil & -0.30 \\
\hline & & & & Peanut butter & -0.29 \\
\hline & & & & Vegetables & -0.28 \\
\hline & & & & Oil fat (not olive oil) & -0.24 \\
\hline \multirow{2}{*}{\multicolumn{6}{|c|}{ Pearson correlation coefficients }} \\
\hline & & & & & \\
\hline Mono- and disaccharides & 0.50 & & 0.87 & & -0.02 \\
\hline Saturated fatty acids & 0.60 & & -0.33 & & 0.72 \\
\hline Total fatty acids & 0.62 & & -0.37 & & -0.69 \\
\hline
\end{tabular}

HSHF dietary pattern, combined high-sugar and high-saturated-fat; HS, high-sugar; HF, high-saturated-fat.

*The presented food groups with absolute factor loadings of $\geq 0.20$ were interpreted as being characteristic for the dietary patterns.

'HF dietary pattern' (Table 2). The explained variation in the response variables was highest for the HSHF dietary pattern (60\%), but was lower in the HS dietary pattern (11\%) and very low in the HF dietary pattern (1\%).

\section{Linear regression analysis between dietary pattern scores and depressive symptoms}

Higher scores on the HSHF dietary pattern were significantly associated with higher depressive symptoms. Due to logarithmic transformation of the PHQ-9 scale score, regression coefficients should be interpreted as follows: in model 3 (fully adjusted model), one-unit increase of the continuous dietary pattern score was related to an increase of $8 \%$ on the PHQ-9 score. When comparing extreme quartiles, an increase of $18 \%$ on the PHQ-9 scores was observed (Table 3). No statistically significant association was observed between continuous scores or when comparing extreme quartiles of the HS dietary pattern or the HF dietary pattern and depressive symptoms in the fully adjusted models (Table 3).

\section{Logistic regression analysis between dietary pattern scores and depressed mood}

We present the association between quartiles and continuous scores of dietary patterns with depressed mood (PHQ-9 sum score $\geq 10$ ) in Table 4 . When we compared extreme quartiles, the HSHF dietary pattern was associated with a 2.36 higher odds of depressed mood. When using continuous dietary pattern scores, one-unit increase of the HSHF dietary pattern was associated with a 1.54 higher odds of depressed mood. Again, no statistically significant association was observed between the HS dietary pattern or the HF dietary pattern and depressed mood after full adjustment for confounders (Table 4).

\section{Sensitivity analysis}

In sensitivity analyses, 749 participants were considered as having extreme energy intakes and were therefore excluded from the analyses. Associations became slightly weaker between the continuous scores of the HSHF dietary pattern and depressive symptoms in all models, but no significant changes were observed (fully adjusted model: $\beta=0 \cdot 07,95 \%$ CI $0 \cdot 01,0 \cdot 12, P=0 \cdot 014)$. Additionally, after full adjustment for confounders, no major changes were observed in the HS dietary pattern $(\beta=-0.01,95 \% \mathrm{CI}-0.06,0.04, P=0.749)$ and the HF dietary pattern $(\beta=-0.02,95 \% \mathrm{CI}-0.07,0.02, P=0.749)$. Finally, we compared extreme quartiles and no changes were observed in the results between any of the dietary patterns and depressive symptoms (data not shown). When we repeated the analyses without extreme energy intakes for depressed mood, again no differences were observed in the continuous scores as well as quartiles in any of the dietary patterns (data not shown).

Furthermore, since the HS dietary pattern revealed high loadings of low-fat dairy products and fruit (high in dietary fibre), we additionally adjusted for fibre intake $(\mathrm{g} / \mathrm{d})$ due to its possible protective effect on depressive symptoms ${ }^{(8)}$. Further adjustments were made by including fruit $(\mathrm{g} / \mathrm{d})$ and low-fat dairy products $(\mathrm{g} / \mathrm{d})$ as confounders. After adjustment for fibre and low-fat dairy intake, no changes were observed in the association, whereas after additional adjustment for fruit intake, the association between the HS dietary pattern and depressive symptoms became positive, but significance was not reached (data not shown).

Finally, RRR analyses were repeated two times by first including mono- and disaccharides as the single response variable $(67 \%$ of the variation was explained by monoand disaccharides) and this dietary pattern was represented by high intakes of sugar-sweetened beverages, 
Table 3 Regression coefficients and $95 \% \mathrm{Cl}$ for the association between the continuous dietary pattern scores and depressive symptoms sum score in the HELIUS (Healthy Life in an Urban Setting) study, Amsterdam, the Netherlands, 2011-2015

\begin{tabular}{|c|c|c|c|c|c|c|c|c|c|}
\hline & \multicolumn{3}{|c|}{ HSHF dietary pattern } & \multicolumn{3}{|c|}{ HS dietary pattern } & \multicolumn{3}{|c|}{ HF dietary pattern } \\
\hline & $\beta^{*}$ & $95 \% \mathrm{Cl}$ & $P$ & $\beta^{*}$ & $95 \% \mathrm{Cl}$ & $P$ & $\beta^{*}$ & $95 \% \mathrm{Cl}$ & $P$ \\
\hline \multicolumn{10}{|c|}{ Dietary pattern scores in quartiles } \\
\hline \multicolumn{10}{|c|}{ Model 1} \\
\hline Q2 & 0.03 & $-0.05,0.10$ & 0.489 & $-0 \cdot 10$ & $-0.18,-0.03$ & 0.005 & -0.01 & $-0.09,0.07$ & 0.720 \\
\hline Q3 & 0.12 & $0.04,0.20$ & 0.004 & $-0 \cdot 10$ & $-0.18,-0.02$ & 0.012 & 0.03 & $-0.06,0.12$ & 0.509 \\
\hline Q4 & 0.20 & $0.09,0.30$ & $<0.001$ & -0.08 & $-0.18,0.02$ & $0 \cdot 101$ & 0.03 & $-0.07,0.13$ & 0.558 \\
\hline \multicolumn{10}{|l|}{ Model 2} \\
\hline Q2 & 0.02 & $-0.05,0.10$ & 0.540 & -0.07 & $-0.14,0.00$ & 0.056 & 0.02 & $-0.06,0.09$ & 0.688 \\
\hline Q3 & 0.12 & $0.04,0.20$ & 0.005 & -0.06 & $0.14,0.02$ & $0 \cdot 124$ & 0.05 & $-0.03,0.14$ & 0.216 \\
\hline Q4 & 0.17 & $0.05,0.28$ & 0.004 & -0.06 & $-0.15,0.04$ & 0.252 & 0.01 & $-0.08,0.11$ & 0.813 \\
\hline \multicolumn{10}{|l|}{ Model 3} \\
\hline Q2 & 0.04 & $-0.03,0.11$ & 0.263 & -0.06 & $-0.13,0.01$ & 0.087 & 0.03 & $-0.05,0.10$ & 0.466 \\
\hline Q3 & 0.14 & $0.06,0.22$ & 0.001 & -0.04 & $-0.12,0.03$ & 0.271 & 0.06 & $-0.02,0.14$ & 0.150 \\
\hline Q4 & 0.18 & $0.07,0.30$ & 0.001 & -0.04 & $-0.13,0.05$ & 0.402 & 0.02 & $-0.08,0.11$ & 0.707 \\
\hline \multicolumn{10}{|c|}{ Continuous dietary pattern scores } \\
\hline Model 1 & 0.07 & $0.04,0.10$ & $<0.001$ & -0.01 & $-0.07,0.04$ & 0.601 & -0.02 & $-0.07,0.02$ & 0.322 \\
\hline Model 2 & 0.07 & $0.02,0.12$ & 0.005 & -0.02 & $-0.07,0.03$ & 0.476 & -0.03 & $-0.07,0.02$ & 0.268 \\
\hline Model 3 & 0.08 & $0.03,0.13$ & 0.002 & -0.01 & $-0.06,0.04$ & 0.771 & -0.02 & $-0.07,0.02$ & 0.306 \\
\hline
\end{tabular}

HSHF dietary pattern, combined high-sugar and high-saturated-fat; HS, high-sugar; HF, high-saturated-fat.

Model 1: adjusted for ethnicity, ethnicity $\times$ dietary pattern score, age and gender.

Model 2: adjusted for model 1 and for marital status, employment status, physical activity and energy intake.

Model 3: adjusted for model 2 and for CVD, diabetes and BMI.

${ }^{*}$ Regression coefficients are log-transformed.

Table 4 Odds ratios and $95 \% \mathrm{Cl}$ for the association between the continuous dietary pattern scores and depressed mood ${ }^{*}$ in the HELIUS (Healthy Life in an Urban Setting) study, Amsterdam, the Netherlands, 2011-2015

\begin{tabular}{|c|c|c|c|c|c|c|c|c|c|}
\hline & \multicolumn{3}{|c|}{ HSHF dietary pattern } & \multicolumn{3}{|c|}{ HS dietary pattern } & \multicolumn{3}{|c|}{ HF dietary pattern } \\
\hline & OR & $95 \% \mathrm{Cl}$ & $P$ & OR & $95 \% \mathrm{Cl}$ & $P$ & OR & $95 \% \mathrm{Cl}$ & $P$ \\
\hline \multicolumn{10}{|c|}{ Dietary pattern scores in quartiles } \\
\hline \multicolumn{10}{|c|}{ Model 1} \\
\hline Q2 & 1.31 & $0.96,1.78$ & 0.086 & 0.83 & $0.61,1.13$ & 0.236 & 0.80 & $0.60,1.07$ & 0.132 \\
\hline Q3 & 1.97 & $1.27,3.06$ & 0.002 & 0.81 & $0.52,1.26$ & 0.341 & 0.80 & $0.52,1.23$ & 0.307 \\
\hline Q4 & $3 \cdot 10$ & $1.72,5.61$ & $<0.001$ & 0.81 & $0.45,1.46$ & 0.492 & 0.90 & $0.50,1.61$ & 0.722 \\
\hline \multicolumn{10}{|l|}{ Model 2} \\
\hline Q2 & $1 \cdot 19$ & $0.86,1.65$ & 0.300 & 0.94 & $0.68,1.30$ & 0.703 & 0.94 & $0.69,1.29$ & 0.712 \\
\hline Q3 & 1.73 & $1.08,2.77$ & 0.023 & 0.95 & $0.60,1.51$ & 0.835 & 0.98 & $0.63,1.53$ & 0.931 \\
\hline Q4 & $2 \cdot 23$ & $1 \cdot 14,4 \cdot 36$ & 0.020 & 0.87 & $0.47,1.59$ & 0.650 & 1.03 & $0.56,1.90$ & 0.915 \\
\hline \multicolumn{10}{|l|}{ Model 3} \\
\hline Q2 & 1.24 & $0.89,1.73$ & 0.201 & 0.95 & $0.69,1.33$ & 0.772 & 0.95 & $0.69,1.30$ & 0.751 \\
\hline Q3 & 1.82 & $1.13,2.94$ & 0.014 & 0.99 & $0.62,1.59$ & 0.983 & 0.95 & $0.60,1.49$ & 0.820 \\
\hline Q4 & 2.36 & $1.19,4.66$ & 0.014 & 0.91 & $0.49,1.67$ & 0.753 & 0.98 & $0.53,1.81$ & 0.943 \\
\hline \multicolumn{10}{|c|}{ Continuous dietary pattern scores } \\
\hline Model 1 & 1.38 & $1.22,1.57$ & $<0.001$ & 1.09 & $0.87,1.38$ & 0.460 & 0.90 & $0.73,1.10$ & 0.288 \\
\hline Model 2 & 1.52 & $1.25,1.85$ & $<0.001$ & 1.03 & $0.82,1.29$ & 0.808 & 0.97 & $0.79,1 \cdot 18$ & 0.739 \\
\hline Model 3 & 1.54 & $1.26,1.88$ & $<0.001$ & 1.05 & $0.83,1.32$ & 0.690 & 0.95 & $0.78,1.16$ & 0.949 \\
\hline
\end{tabular}

HSHF dietary pattern, combined high-sugar and high-saturated-fat; HS, high-sugar; HF, high-saturated-fat; PHQ-9, nine-item Patient Health Questionnaire. Model 1: adjusted for ethnicity, ethnicity $\times$ dietary pattern score, age and gender.

Model 2: adjusted for model 1 and for marital status, employment status, physical activity and energy intake.

Model 3: adjusted for model 2 and for CVD, diabetes and BMI.

*Depressed mood is defined as PHQ-9 sum score $\geq 10$.

natural fruit juices, added sugars, chocolates, sweets and pastries, low- and high-fat dairy products, fruit and savoury snacks. Second, we included saturated fatty acids as the single response variable and a dietary pattern high in red meat, chocolates, sweets and pastries, high-fat dairy products, butter, pasta, potatoes and fast foods was identified $(71 \%$ of the variation was explained by saturated fatty acids). Overall, both dietary patterns resembled high intakes of food groups that were both high in sugar and saturated fat and were therefore similar to the HSHF dietary pattern from our main analyses. In other words, we were not able to obtain a dietary pattern that distinguished between sugar and saturated fat when including a single response variable.

\section{Discussion}

In the present study, we investigated whether consuming an HS dietary pattern, an HF dietary pattern or an HSHF 
dietary pattern was associated with depressive symptoms among a multi-ethnic population living in Amsterdam, the Netherlands. We found that consumption of the HSHF dietary pattern was associated with significantly higher depressive symptoms and depressed mood. In contrast, no significant associations were found between consumption of either the HS dietary pattern or the HF dietary pattern and depressive symptoms or depressed mood.

Our findings suggest that a dietary pattern containing high-sugar and high-saturated-fat foods increases depressive symptoms. A plausible mechanism for this increase in depressive symptoms is that an HSHF dietary pattern, which is close to a Western dietary pattern, might be related to higher inflammatory levels in the body and CHD, which in turn are both indicated to increase depressive symptoms ${ }^{(9,10,23,41)}$. To our knowledge, the present study is the first that has (i) analysed a combined high-sugar and high-saturated-fat dietary pattern that was identified by RRR and (ii) evaluated the separate effect of sugar and saturated fat in one study in relation to depressive symptoms and depressed mood in a multiethnic population.

Previous studies investigated Western dietary patterns that were high in both sugar and saturated fat in relation to depressive symptoms, of which three studies found a significant positive relationship between a Western dietary pattern and depressive symptoms ${ }^{(9-11)}$, whereas three other studies observed a positive relationship between a Western dietary pattern and depressive symptoms, although not statistically significant ${ }^{(12-14)}$. These differences in findings may be explained by differences in the measurement of dietary intake and depressive symptoms $^{(7)}$.

By using RRR, we were able to obtain dietary patterns that distinguished between high-sugar and high-saturatedfat food groups; however, we found no association between either the HS dietary pattern or the HF dietary pattern and depressive symptoms or depressed mood. Both these dietary patterns included a restricted number of foods and one may argue whether these food groups reflect a dietary pattern. Furthermore, both dietary patterns explained a small variation of the total food intake (explained variation of the HS dietary pattern was $11 \%$ while the explained variation of the HF dietary pattern was only $1 \%$ ), although the explained variation of $11 \%$ is quite common while the first factor usually explains the highest amount of variation in the response variables compared with the remaining extracted factors ${ }^{(26)}$. Our findings reflect that it is impractical to distinguish the contribution of individual macronutrients to the association between diet and health because most foods, especially processed foods, are often high in sugar as well as in saturated fat. This was confirmed in our sensitivity analysis in which we conducted RRR using only mono- and disaccharides as the response variable and afterwards only saturated fatty acids as the response variable. In both cases we identified a dietary pattern with food groups that are high in both sugar and saturated fat. Furthermore, extreme energy intake did not seem to explain the association between dietary patterns and depressive symptoms since no substantial changes were observed for the association between any of the dietary patterns and depressive symptoms after we excluded participants with extreme energy intakes.

Due to differences in macronutrient intakes across ethnic groups in the Netherlands (Surinamese consume more sugar whereas Dutch consume more foods that are high in both sugar and saturated fat ${ }^{(27,38,42,43)}$, heterogeneity is present. The high variety of dietary intakes in these ethnic groups can be an advantage because with this heterogeneity we can capture the more extreme intakes of various diets. Thereby we were able to differentiate between dietary patterns with diverse macronutrient compositions. This could be considered a strength of our study. Using RRR to derive dietary patterns could be considered another strength of the study. By using RRR, we were able to investigate the independent contributions of different nutrients in overall dietary patterns that are closely related to our outcome measure and which are representative for our study population.

Several limitations need to be acknowledged as well. Due to the cross-sectional design of the current study, no causal inferences can be made and reverse causation cannot be ruled out. More specifically, it might be that participants who are depressed eat more unhealthy foods that are both high in sugar and saturated fat, so depression may cause unhealthy eating and not the other way around $^{(5)}$. Furthermore, FFQ are memory-based self-report questionnaires. People tend to under-report unhealthy food items which in turn may lead to lower observed energy intakes. Thereby, the observed association is likely to be underestimated. Another limitation is that we did not use a clinical diagnosis of depression but the subjective PHQ-9 questionnaire. A previous study observed a sensitivity of $88 \%$ (when using a cut-off point of $\geq 10)^{(33)}$, which indicates that $12 \%$ of depression cases may go undetected. However, the PHQ-9 has shown to be a valid screening instrument to measure depressive symptoms previously in different ethnic populations ${ }^{(34,35,44)}$.

\section{Implications of findings}

Based on these results, it can be indicated that the consumption of high-sugar and high-saturated-fat foods (as part of the HSHF dietary pattern) should be replaced by more nutrient-dense foods such as wholegrain products, fatty fish, fruit and vegetables, to lower depressive symptoms. The high levels of antioxidants (fruit and vegetables), fibre (whole grains) and $n$ - 3 fatty acids (fatty fish) in these foods may lower oxidative stress levels as well as inflammation, which in turn may decrease depression $^{(41,45)}$. Thereby a healthy dietary pattern could 
act as a health promotor in relation to depressive symptoms. Although these recommendations are the same for all ethnic groups, the intake of ethnic-specific foods, as part of the dietary patterns, may differ among ethnic groups and should be taken into account in dietary guidelines.

\section{Conclusion}

In this multi-ethnic population, higher consumption of a HSHF dietary pattern (high loadings of chocolates, sweets and pastries, red meat, added sugars, high-fat dairy products, fried foods, creamy sauces) was associated with higher depressive symptoms and depressed mood, whereas no association was found for either the HS dietary pattern or the HF dietary pattern. Above all, our findings reinforce the idea that it is not attainable to disentangle the effects of single macronutrients in studying diet and disease relationships. Therefore, no clear conclusions can be drawn concerning the independent contribution of an HS dietary pattern or an HF dietary pattern in relation to depressive symptoms. Additionally, it is important for future studies to focus on overall dietary patterns containing foods that are high in both sugar as well as saturated fat, instead of focusing on separate macronutrients. However, the results need to be interpreted with caution due to the cross-sectional nature of the study and therefore longitudinal studies and experimental trials are needed to confirm these results.

\section{Acknowledgements}

Acknowledgements: The authors are most grateful to the participants of the HELIUS study and the management team, research nurses, interviewers, research assistants and other staff who have taken part in gathering the data for this study. Financial support: The HELIUS study is conducted by the Academic Medical Center Amsterdam and the Public Health Service of Amsterdam. Both organizations provided core support for HELIUS. The HELIUS study is also funded by the Dutch Heart Foundation, the Netherlands Organization for Health Research and Development (ZonMw), the European Union (FP7) and the European Fund for the Integration of non-EU immigrants (EIF). This work was additionally supported by the EU FP7 MooDFOOD Project 'Multi-country cOllaborative project on the rOle of Diet, FOod-related behaviour, and Obesity in the prevention of Depression' (grant number 613598). The funders had no role in the design, analysis or writing of this article. Conflict of interest: None. Authorship: The authors' responsibilities were as follows. E.V.: analytic design, data analysis, manuscript preparation and had primary responsibility for final content; M.N.: analytic design, interpretation of the results and critical review of the manuscript; K.S., M.V. and I.A.B.: interpretation of the results and critical review of the manuscript; M.B.S., A.H.S., A.L. and J.H.d.V.: critical review of the manuscript. All authors have read and approved the final manuscript. Ethics of human subject participation: All study protocols were approved by the Academic Medical Centre Ethical Review Board, and all participants provided written informed consent.

\section{Supplementary material}

To view supplementary material for this article, please visit https://doi.org/10.1017/S1368980017001550

\section{References}

1. Marcus M, Yasamy MT, van Ommeren M et al. (2012) Depression: A Global Public Health Concern. http://www. who.int/mental_health/management/depression/who_paper_ depression_wfmh_2012.pdf (accessed June 2017).

2. Verweij $G$ \& Houben-van Harten M (2013) Depressiviteit en antidepressiva in Nederland (Depression and AntiDepression Medication in the Netherlands). Den Haag: Centraal Bureau voor de Statistiek.

3. Ikram UZ, Snijder MB, Fassaert TJ et al. (2015) The contribution of perceived ethnic discrimination to the prevalence of depression. Eur J Public Health 25, 243-248.

4. Martinez-Gonzalez MA \& Sanchez-Villegas A (2016) Food patterns and the prevention of depression. Proc Nutr SoC 75, 139-146.

5. Sanchez-Villegas A \& Martinez-Gonzalez MA (2013) Diet, a new target to prevent depression? BMC Med 11, 3 .

6. Jacka FN, O'Neil A, Opie R et al. (2017) A randomised controlled trial of dietary improvement for adults with major depression (the 'SMILES' trial). BMC Med 15, 23.

7. Lai JS, Hiles S, Bisquera A et al. (2014) A systematic review and meta-analysis of dietary patterns and depression in community-dwelling adults. Am J Clin Nutr 99, 181-197.

8. Miki T, Eguchi M, Kurotani K et al. (2016) Dietary fiber intake and depressive symptoms in Japanese employees: the Furukawa Nutrition and Health Study. Nutrition 32, 584-589.

9. Akbaraly TN, Brunner EJ, Ferrie JE et al. (2009) Dietary pattern and depressive symptoms in middle age. $\mathrm{Br} J$ Psychiatry 195, 408-413.

10. Le Port A, Gueguen A, Kesse-Guyot E et al. (2012) Association between dietary patterns and depressive symptoms over time: a 10-year follow-up study of the GAZEL cohort. PLoS One 7, e51593.

11. Jacka FN, Pasco JA, Mykletun A et al. (2010) Association of Western and traditional diets with depression and anxiety in women. Am J Psychiatry 167, 305-311.

12. Jacka FN, Mykletun A, Berk M et al. (2011) The association between habitual diet quality and the common mental disorders in community-dwelling adults: the Hordaland Health study. Psychosom Med 73, 483-490.

13. Chocano-Bedoya PO, O'Reilly EJ, Lucas M et al. (2013) Prospective study on long-term dietary patterns and incident depression in middle-aged and older women. $\mathrm{Am} \mathrm{J}$ Clin Nutr 98, 813-820.

14. Rienks J, Dobson AJ \& Mishra GD (2013) Mediterranean dietary pattern and prevalence and incidence of depressive symptoms in mid-aged women: results from a large community-based prospective study. Eur J Clin Nutr 67, 75-82.

15. Sanchez-Villegas A, Verberne L, De Irala J et al. (2011) Dietary fat intake and the risk of depression: the SUN Project. PLoS One 6, e16268. 
16. Westover AN \& Marangell LB (2002) A cross-national relationship between sugar consumption and major depression? Depress Anxiety 16, 118-120.

17. Jeffery RW, Linde JA, Simon GE et al. (2009) Reported food choices in older women in relation to body mass index and depressive symptoms. Appetite 52, 238-240.

18. Mwamburi DM, Liebson E, Folstein M et al. (2011) Depression and glycemic intake in the homebound elderly. $J$ Affect Disord 132, 94-98.

19. Gangwisch JE, Hale L, Garcia L et al. (2015) High glycemic index diet as a risk factor for depression: analyses from the Women's Health Initiative. Am J Clin Nutr 102, 454-463.

20. Siri-Tarino PW, Chiu S, Bergeron N et al. (2015) Saturated fats versus polyunsaturated fats versus carbohydrates for cardiovascular disease prevention and treatment. Annu Rev Nutr 35, 517-543.

21. Jakobsen MU, Dethlefsen C, Joensen AM et al. (2010) Intake of carbohydrates compared with intake of saturated fatty acids and risk of myocardial infarction: importance of the glycemic index. Am J Clin Nutr 91, 1764-1768.

22. Poole L, Dickens C \& Steptoe A (2011) The puzzle of depression and acute coronary syndrome: reviewing the role of acute inflammation. J Psychosom Res 71, 61-68.

23. Valkanova V \& Ebmeier KP (2013) Vascular risk factors and depression in later life: a systematic review and metaanalysis. Biol Psychiatry 73, 406-413.

24. Ambrosini GL, Emmett PM, Northstone K et al. (2012) Identification of a dietary pattern prospectively associated with increased adiposity during childhood and adolescence. Int J Obes (Lond) 36, 1299-1305.

25. Hoffmann K, Schulze MB, Schienkiewitz A et al. (2004) Application of a new statistical method to derive dietary patterns in nutritional epidemiology. Am J Epidemiol 159, 935-944.

26. Weikert C \& Schulze MB (2016) Evaluating dietary patterns: the role of reduced rank regression. Curr Opin Clin Nutr Metab Care (Epublication ahead of print version).

27. Brussaard JH, van Erp-Baart MA, Brants HA et al. (2001) Nutrition and health among migrants in The Netherlands. Public Health Nutr 4, 659-664.

28. de Boer EJ, Brants HAM, Beukers M et al. (2015) Voeding van Marokkaanse, Turkse, Surinaamse en autochtone Nederlanders in Amsterdam. Bilthoven: Rijksinstituut voor Volksgezondheid en Milieu.

29. Dijkshoorn H, Nierkens V \& Nicolaou M (2008) Risk groups for overweight and obesity among Turkish and Moroccan migrants in The Netherlands. Public Health Nutr 122, 625-630.

30. Stronks K, Snijder MB, Peters RJ et al. (2013) Unravelling the impact of ethnicity on health in Europe: the HELIUS study. BMC Public Health 13, 402.

31. Simon P (2012) Collecting ethnic statistics in Europe: a review. Ethn Racial Stud 35, 1366-1391.

32. Dekker LH, Snijder MB, Beukers MH et al. (2011) A prospective cohort study of dietary patterns of non-western migrants in the Netherlands in relation to risk factors for cardiovascular diseases: HELIUS-Dietary Patterns. BMC Public Health 11, 441.

33. Kroenke K, Spitzer RL \& Williams JB (2001) The PHQ-9: validity of a brief depression severity measure. J Gen Intern Med 16, 606-613.

34. Manea L, Gilbody S \& McMillan D (2015) A diagnostic metaanalysis of the Patient Health Questionnaire-9 (PHQ-9) algorithm scoring method as a screen for depression. Gen Hosp Psychiatry 37, 67-75.

35. Wittkampf KA, Naeije L, Schene AH et al. (2007) Diagnostic accuracy of the mood module of the Patient Health Questionnaire: a systematic review. Gen Hosp Psychiatry 29, 388-395.

36. Beukers MH, Dekker LH, de Boer EJ et al. (2015) Development of the HELIUS food frequency questionnaires: ethnicspecific questionnaires to assess the diet of a multiethnic population in The Netherlands. Eur J Clin Nutr 69, 579-584.

37. National Institute for Public Health \& the Netherlands Nutrition Centre (2011) Dutch Food Composition Table 2011. The Hague: National Institute for Public Health and the Netherlands Nutrition Centre.

38. van Dam R \& Nicolau M (2005) Voedingspatroon van Surinaamse Amsterdammers in kaart gebracht. Nederlands Tijdschrift voor Diëtisten 60, 98-102.

39. Goldberg GR, Black A, Jebb SA et al. (1991) Critical evaluation of energy intake data using fundamental principles of energy physiology: 1. Derivation of cut-off limits to identify under-recording. Eur J Clin Nutr 45, 569-581.

40. Black AE (2000) Critical evaluation of energy intake using the Goldberg cut-off for energy intake:basal metabolic rate. A practical guide to its calculation, use and limitations. Int $J$ Obes Relat Metab Disord 24, 1119-1130.

41. Lopez-Garcia E, Schulze MB, Fung TT et al. (2004) Major dietary patterns are related to plasma concentrations of markers of inflammation and endothelial dysfunction. Am J Clin Nutr 80, 1029-1035.

42. Palsma AH NM, Van Dam RM \& Stronks K (2006) De voeding van Turkse en Marokkaanse Nederlanders in de leeftijd van 18-30 jaar: prioriteiten voor voedingsinterventies. Tijdschrift voor Gezondheidswetenschappen 85, 415-422.

43. Rijksinstituut voor Volksgezondheid en Milieu, Centrum voor Voeding en Gezondheid (2007-2010) VCP-Basis 7-69 jaar, 2007-2010. http://www.rivm.nl/Onderwerpen/V/Voedsel consumptiepeiling/Overzicht_voedselconsumptiepeilingen/ VCP_Basis_7_69_jaar_2007_2010 (accessed March 2011).

44. Baas KD, Cramer AO, Koeter MW et al. (2011) Measurement invariance with respect to ethnicity of the Patient Health Questionnaire-9 (PHQ-9). J Affect Disord 129, 229-235.

45. Sarandol A, Sarandol E, Eker SS et al. (2007) Major depressive disorder is accompanied with oxidative stress: short-term antidepressant treatment does not alter oxidative-antioxidative systems. Hum Psychopharmacol 22, 67-73. 